\title{
Micromolding for three-dimensional metal microstructures using stereolithography of photopolymerized resin
}

\author{
Kohki Mukai, Shinya Kitayama, Yasunobu Kawajiri, Shoji Maruo \\ Department of Mechanical Engineering and Materials Science, \\ Yokohama National University, \\ 79-5 Tokiwadai, Hodogaya-Ku, Yokohama, Kanagawa 240-0197, Japan \\ Tel \& Fax: +81-45-339-3853, email: mukai@ynu.ac.jp
}

\begin{abstract}
If micromachines can be produced with metal microstructures, their application field will be greatly expanded. We have proposed a metal micromolding method based on the stereolithography of photopolymerized resin. In this work, we report our recent successful development of three fundamental processes used in the micromolding method. The electroless plating of palladium with a purity of $93-97 \%$ was realized on photopolymerized resin with a view to realizing a shape memory alloy. An electrolytic grinding technique was examined for copper, and step-by-step grinding was realized for the first time using a $\mathrm{NaCl}$ solution. A copper replica was successfully extracted from a resin mold by using heat treatment. The results suggested that three-dimensional metal microstructures can be produced with micrometer order accuracy by using the micromolding method.
\end{abstract}

Keywords: micromolding; microstructure; photopolymerized resin; stereolithography; micromachine 


\section{Introduction}

Micro electro mechanical systems (MEMS) are now widely employed. However, most of their main components are made of semiconductors, and metal is not used in the body because it is difficult to micro-process. If the special properties of metal, such as magnetism, high conductivity, and the shape memory effect were available, the MEMS application field would be greatly expanded. In addition, MEMS has a twodimensional-layer structure since it is produced by using sacrificial layer etching. If complicated three-dimensional structures could be adopted for MEMS, the application field would be even further expanded. Microfabrication with photopolymerized resin is an attractive and well-known technology [1 - 3]. It enables complicated threedimensional structures to be manufactured without large-scale equipment. However, resin structures are not tolerant to chemical and heat treatment and this limits their application. Metal plating the resin is one method of overcoming this problem $[4,5]$. However, this method does not solve the problem completely since the resin still remains inside the structure.

We have proposed and developed a micromolding method for the production of pure-metal three-dimensional microstructures [6 - 9]. This method consists of four main steps (Figure 1). First, raw resin is polymerized by scanning a focused laser beam to form the three-dimensional negative shape of a microstructure with a small opening. The resolution of this polymerization is now better than $30 \mathrm{~nm}$ with the two-photon absorption method [10]. In the next step, the non-conductive polymer mold is coated with metal using electroless plating. At this stage, the inner space of the negative shape is filled with metal. We have succeeded in plating nickel, copper, and ferrite on resin suitable for two-photon polymerization. Microstructures will be provided with multi 
functions by making different kinds of metal multilayer. In the third step, the obstructive metal is removed by electrolytic grinding to expose the opening of the mold. The electrolytic grinding stops automatically when the non-conductive resin is exposed and the inner metal microstructure and outer metal coating are electrically isolated [11]. Therefore, a precise cut is achieved at the opening. In the final step, the microstructure is extracted by removing the resin mold. We have already reported the chemical removal technique.

In this work, we describe the successful development of three steps in the micromolding method: electroless plating, electrolytic grinding, and extraction. We examined the electroless plating of the photopolymerized resin with palladium. We realized the electrolytic grinding of copper. We extracted a metal replica from a resin mold using a heat treatment.

\section{Electroless Palladium Plating}

We studied the electroless plating of palladium on a photopolymer surface. Palladium is very attractive in terms of MEMS applications because a palladium-iron alloy exhibits the shape memory effect. Once electroless palladium plating is successfully carried out with a resin mold, a palladium-iron alloy microstructure will be producible when the well-known iron electrolytic plating technique is employed with palladium to be alloyed. In the experiments, first a cup made of silicone was prepared, and raw resin suitable for two-photon polymerization was cured in the cup. To simplify the experiment, an ultraviolet lamp was used for the polymerization. Finally, a pelletshaped resin base was prepared. Before plating the resin base, we washed it in water and acetone with ultrasonic waves. After washing and catalysis, the resin base was 
immersed in the plating solution. The composition of the catalytic solution was $0.8 \mathrm{~g} / \mathrm{L}$ $\mathrm{PdCl}_{2}+15 \mathrm{~g} / \mathrm{L} \mathrm{SnCl} l_{2}+150 \mathrm{~mL} / \mathrm{L} \mathrm{HCl}$. The composition of the plating solution was 1.8 $\mathrm{g} / \mathrm{L} \mathrm{PdCl}_{2}+60 \mathrm{mg} / \mathrm{L} \mathrm{NH} \mathrm{CH}_{2} \mathrm{CH}_{2} \mathrm{NH}_{2}+3.2 \mathrm{~g} / \mathrm{L} \mathrm{NaH_{2 }} \mathrm{PO}_{2} \cdot \mathrm{H}_{2} \mathrm{O}+30 \mathrm{mg} / \mathrm{L}$ $\mathrm{HSCH}_{2} \mathrm{COOH}$. $\mathrm{HCl}$ was used to control the $\mathrm{pH}$ of the plating solution. We found that the electroless palladium plating was successful when the plating solution temperature was $50^{\circ} \mathrm{C}$.

We investigated the characteristics of the plated films by controlling the $\mathrm{pH}$ of the plating solution. Figure 2 shows the relationship between $\mathrm{pH}$ and the growth rate of the plated film. The thickness of the plated film was evaluated by observing a sample crosssection with a high-resolution optical microscope [9]. The growth rate was defined as the average value measured at four points, and we confirmed that the values were almost constant for 5 hours. The growth rate increased monotonously as the $\mathrm{pH}$ increased from 6 to 12 . The microscope observation also revealed that there were no problems as regards adhesion between the resin and the plated film in this $\mathrm{pH}$ range. The inset shows the appearance of the plated samples. A metallic luster was seen on the sample surface at $\mathrm{pH}$ values of $6-10$. With a $\mathrm{pH}$ value of 12 , the plated film became very uneven and the metallic luster was ambiguous. It appears that minute pieces of film generated in the rapid chemical reaction adhered to the sample surface.

X-ray fluorescence measurement was used to evaluate the components of the plated film. The results are shown in Table I. We can see that the purity of the palladium on the plated film increased from 89.6 to 97.1 as the $\mathrm{pH}$ increased from 6 to 12. The main impurity, $\mathrm{P}$, originates in the plating solution. It is obviously desirable to increase the $\mathrm{pH}$ to 12 in terms of achieving high purity. However, as seen in Figure 2, a 
$\mathrm{pH}$ of 12 is not necessarily preferable with respect to metallic luster, which is related to the homogeneity of the plating. This homogeneity should be considered in connection with the size of the components being produced. The results suggest that high purity can be achieved by increasing the $\mathrm{pH}$ when making small components.

\section{Electrolytic Grinding of Copper Film}

We investigated the electrolytic grinding of copper. The principle of electrolytic grinding is presented in Figure 3(a). The cathode consists of a substance to which the non-conductive grinder adheres with the conductive binder. A resin mold coated with metal is connected to the anode. When current is passed through a circuit while the sample is immersed in a specific electrolysis solution, the electrochemical reaction produces non-conductive film on the surface as the electrolysis output. Then, the current does not flow and the electrochemical reaction stops. Next, the non-conductive film is gently removed with a non-conductive grinder. Then, a current is again supplied. In the micromolding process, the electrolytic grinding stops completely once the resin is exposed.

We first investigated the most suitable electrolysis solution for the electrochemical process. The non-conductive film must completely cover the copper surface so that it halts the electrochemical reaction. We examined three solutions, i.e., $\mathrm{H}_{3} \mathrm{PO}_{4}, \mathrm{NaNO}_{3}$, and $\mathrm{NaCl}$. These solutions are known to realize a non-conductive output with copper electrochemically. We investigated the current versus voltage characteristic with copper plates immersed in these electrolysis solutions, and found that the current flow was prevented with an increase in voltage only in the $\mathrm{NaCl}$ solution. 
The electrochemical reactions are $\mathrm{Cu}+\mathrm{Cl}^{-} \rightarrow \mathrm{CuCl}+\mathrm{e}$ and $\mathrm{CuCl}+\mathrm{Cl}^{-} \rightarrow \mathrm{CuCl}_{2}+$ e. Scanning electron microscopy (SEM) observations revealed many voids on the surface of the electrochemical output when using $\mathrm{H}_{3} \mathrm{PO}_{4}$ and $\mathrm{NaNO}_{3}$ solutions. These voids are expected to cause current leakage.

We succeeded in the step-by-step electrolytic grinding of a copper plate using $\mathrm{NaCl}$ solution under a constant current condition. The relationship between voltage and time is shown in Figure 3(b), where the cyclic grinding process appears clearly. In the experiment, the step-by-step grinding proceeded as follows. First, a non-conductive output covered the surface of the copper plate as a result of the electrochemical reaction. Accordingly, the resistance and voltage both increased. Then, the non-conductive output was removed gently with the grinder. The resistance decreased, and the voltage fell quickly almost to zero. The inset shows the copper plate after the grinding process. Electrolytic grinding was performed on the lower half of the plate, where the color had become greenish black.

Microscope observations were performed to evaluate the non-conductive output. Figure 4(a) shows a bird's-eye view obtained with a laser-scanning microscope near the edge of the non-conductive film on the copper plate after the electrochemical reaction. The right half of the image shows a non-conducting film, which is thicker than the copper plate. Figure 4(b) shows the height profile at the dashed line seen in Figure 4(a). The height increases steeply to about $20 \mu \mathrm{m}$ at the non-conductive film. The thickness fluctuation was as large as $10 \mu \mathrm{m}$. Figure 5(a) and (b), respectively, show SEM images of a cross-section and a plan view of another sample with non-conductive film,. The large surface roughness of the non-conductive film appears again in the figures. By using a very even grindstone, the surface flatness can be corrected during the grinding 
process. In contrast, the homogeneity of the reaction frontier inside the metal is important because it determines the cut shape of the metallic parts. In Figure 5(a), we can see that the reaction frontier exhibits a micrometer order fluctuation. The result suggests that uneven micrometer order corrosion will remain in the copper at the opening of the mold.

\section{Extraction from Resin Mold via Heat Treatment}

We investigated the thermal extraction of a metal structure from a resin mold. For this purpose, we replicated the shape of a screw using copper. Figure 6(a) shows the sample transition during this process. To fabricate the resin mold, we dipped half of a stainless steel screw into a silicone cup filled with raw resin, and irradiated it with an ultraviolet lamp. After polymerization, the original screw was removed by force. Finally, a resin mold with a negative screw shape was prepared. The mold was slightly deformed owing to the forcible removal. Subsequently, the resin mold was plated with copper via the electroless process.

The copper replica was extracted from the resin mold by using heat treatment. First, a circular cut was made along the sidewall of the plated sample to expose the resin to the outside atmosphere. Then, the sample was placed in a furnace and heated at $500^{\circ} \mathrm{C}$ for 20 minutes under a $\mathrm{N}_{2}$ gas flow. Because the oxygen in the furnace was not completely eliminated, the resin was burned away and the ash adhered to the sample surface. Figure 6(b) and (c) show SEM observations of the extracted copper replica after ultrasonic washing had been carried out with organic solvent. Figure 6(a) shows that the screw thread was slightly deformed probably because the mold was already 
deformed by the forcible removal of the original screw. We also found that the replica surface was uneven. This characteristic is clearly revealed in Figure 6(b), where we can see several-micrometers' unevenness and also unevenness of less than a micrometer. We assume that both levels of unevenness originated from the mold, which was prepared by tearing the metal screw from the sticky resin. This surface unevenness will not be a problem when the mold is produced directly by laser beam scanning.

\section{Conclusion}

We investigated three steps in the micromolding process used for the production of three-dimensional metal microstructures, i.e., electroless plating, electrolytic grinding, and extraction. The electroless plating of photopolymerized resin with palladium was examined with a view to realizing a shape memory alloy. We found that palladium film with a purity of $93-97 \%$ can be plated in a $\mathrm{pH}$ range of $10-12$ at $50^{\circ} \mathrm{C}$. Copper electrolytic grinding was examined, and step-by-step grinding was realized for the first time using a $\mathrm{NaCl}$ solution. We showed that fluctuations at the reaction frontier of the non-conductive output will limit the opening accuracy to micrometers. The extraction of a copper replica from a resin mold was also examined. A copper replica of the screw shape was successfully extracted by removing the resin with 20 minutes of heat treatment at $500^{\circ} \mathrm{C}$, These research findings constitute progress in the manufacture technology of three-dimensional metal microstructures. 


\section{References}

[1] S. Maruo and S. Kawata: J. Microelectromech. Syst. 7 (1998) 411.

[2] L. Wu, Y. Zhong, C. T. Chan, K. S. Wong, and G. P. Wang: Appl. Phys. Lett. 86 (2005) 241102.

[3] S. Kawata, H.-B. Sun, T. Tanaka, and K. Takada: Nature 412 (2001) 697.

[4] K. Nishimura, H. Uchida, M. Inoue, M. Sendoh, K. Ishiyama, and K. I. Arai: J. Appl. Phys. 93 (2003) 6712.

[5] F. Formanek, N. Takeyasu, T. Tanaka, K. Chiyoda, A. Ishikawa, and S. Kawata: Appl. Phys. Lett. 88 (2006) 083110.

[6] K. Mukai, T. Yoshimura, S. Maruo, Jpn. J. Appl. Phys. 46 (2007) 2761-2763.

[7] K. Mukai, T. Yoshimura, S. Kitayama, S. Maruo, J. Photopolym. Sci. Technol. 20 (2007) 285.

[8] K. Mukai, S. Kitamura, T. Yoshimura, S. Maruo, Jpn. J. Appl. Phys. 47 (2008) $3232-3235$.

[9] K. Mukai, S. Kitayama, S. Maruo, J. Photopolym. Sci. Technol. 21 (2008) 53-58.

[10] S. H. Park, T. W. Lim, D.-Y. Yang, N. C. Cho and K.-S. Lee, Appl. Phys. Lett. 89 (2006) 173133.

[11] T. Sato, "Denkaikakou to Kagakukakou (Electrolytic Processing and Chemical Processing)" (Asakura Shoten, Tokyo, 1970) (in Japanese).

\section{Figure captions}

Figure 1. The four steps of the micromolding method using stereolithography with photopolymerized resin. 
Figure 2. The relationship between the plating solution $\mathrm{pH}$ and the plated film growth rate. The inset shows the appearance of the resin samples after electroless palladium plating.

Figure 3. (a) Schematic of electrolytic grinding. (b) Voltage versus time characteristics during copper electrolytic grinding. The inset shows the copper plate after the electrolytic grinding cycles.

Figure 4. Laser scanning microscope observation: (a) a bird's-eye view near the edge of the non-conductive film, and (b) height profile at the dashed line in (a).

Figure 5. SEM images of (a) a cross-section and (b) a plan view of samples with the non-conductive film.

Figure 6. (a) Original screw, negative replica (mold) made of resin, and the mold after electroless copper plating. (b) SEM image of the copper replica, and (c) a close-up view of its surface.

Table I. Components of the plated film evaluated by X-ray fluorescence measurements. UDL: under detection limit. 
1 : Photopolymerization
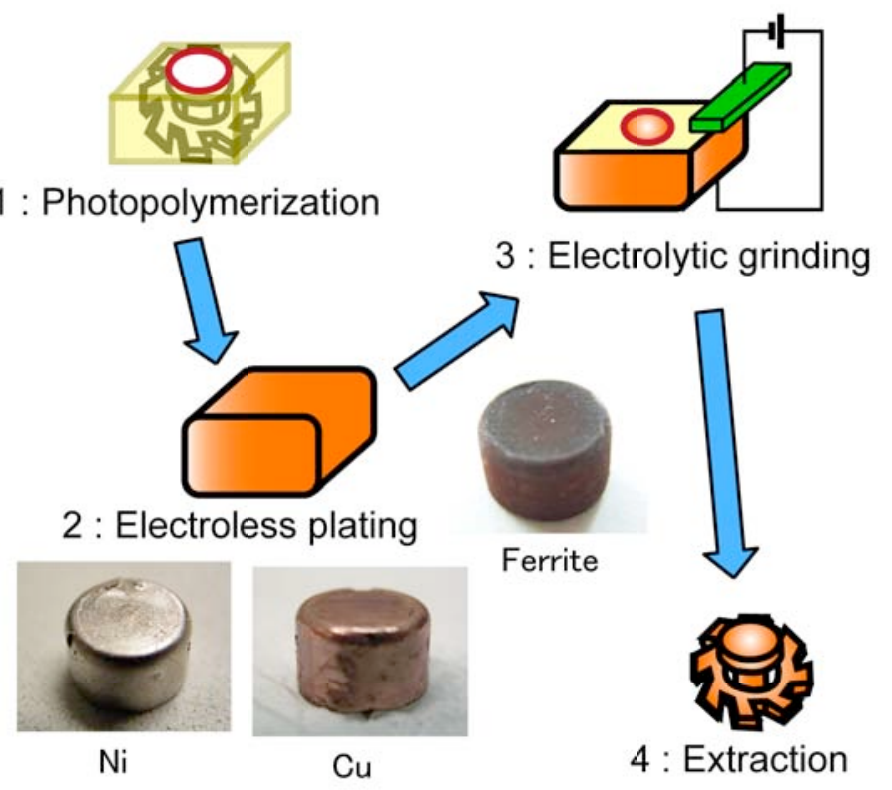

Figure 1 


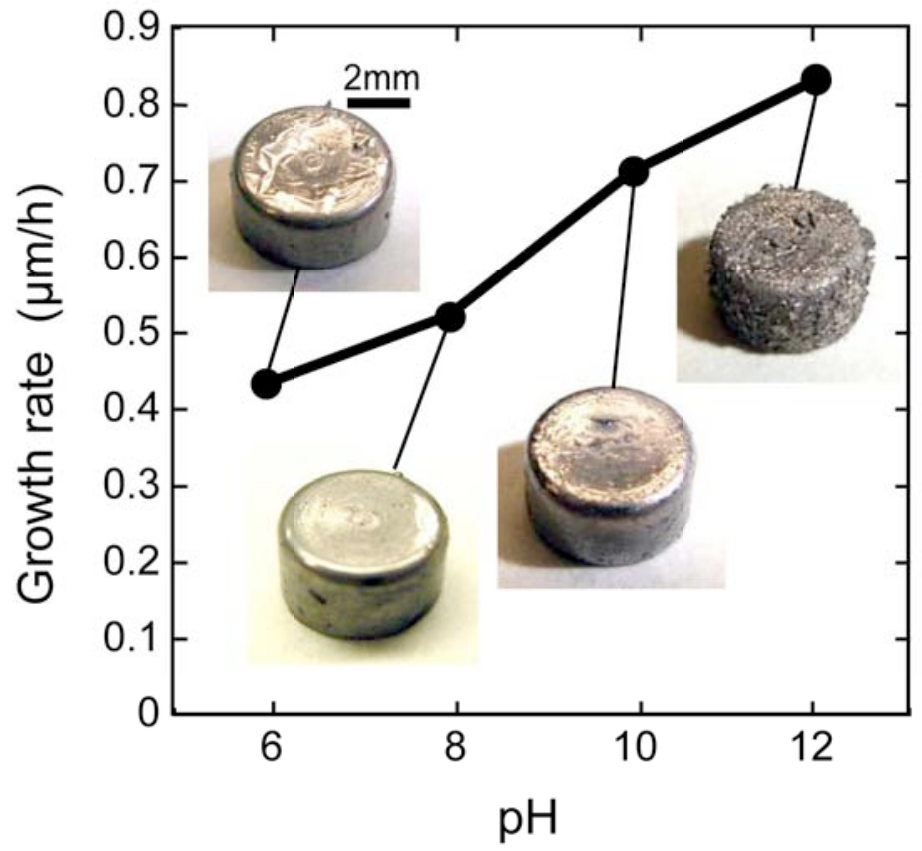

Figure 2 


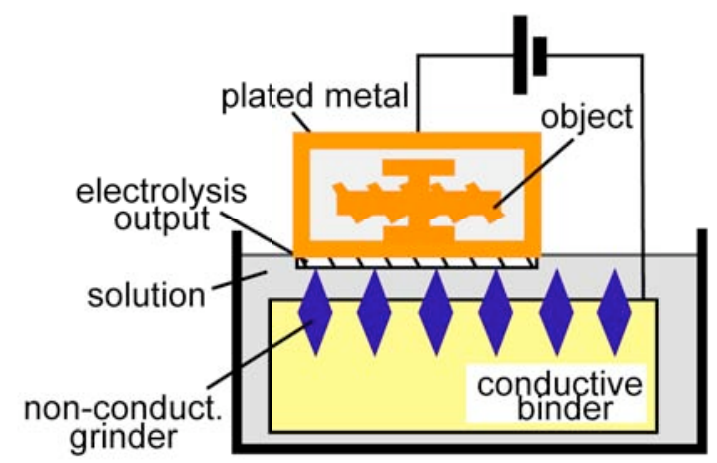

(a)

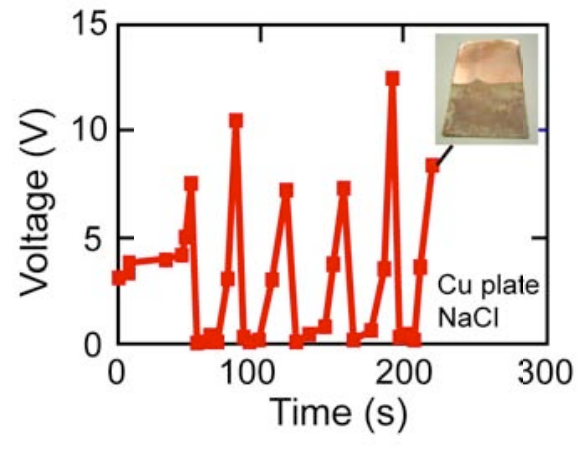

Figure 3 


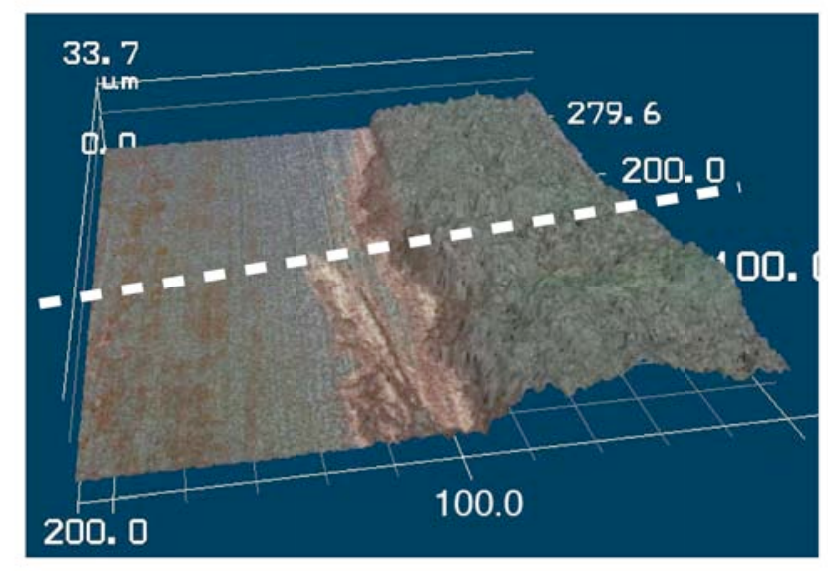

(a)

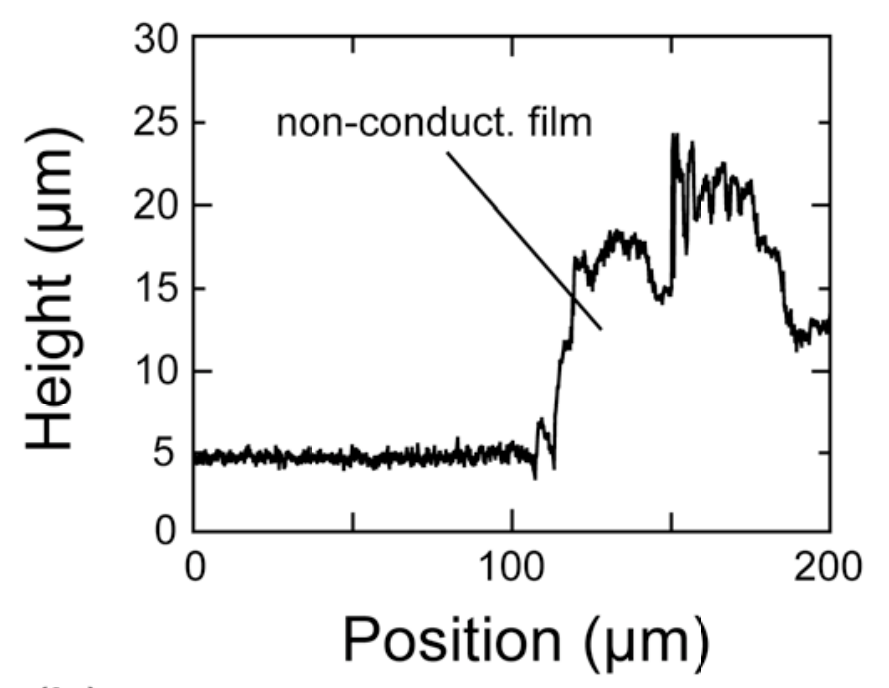

(b)

Figure 4 


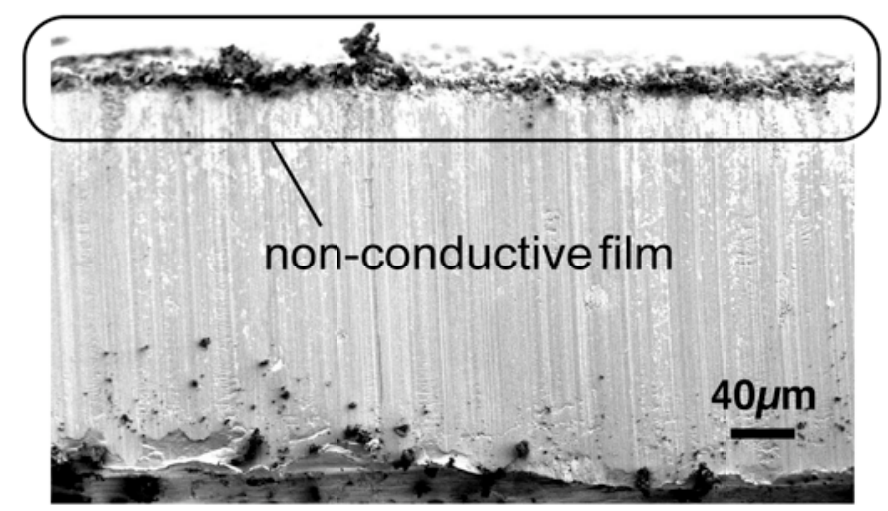

(a)

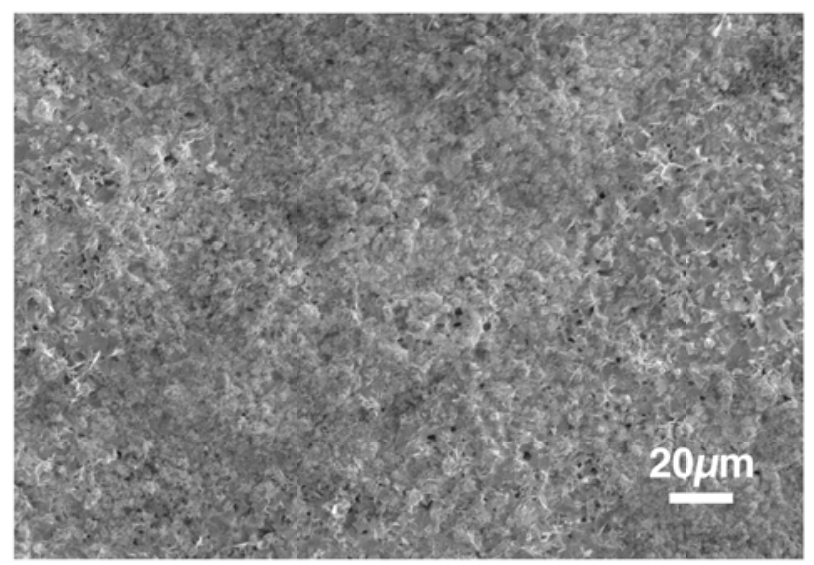

(b)

Figure 5 


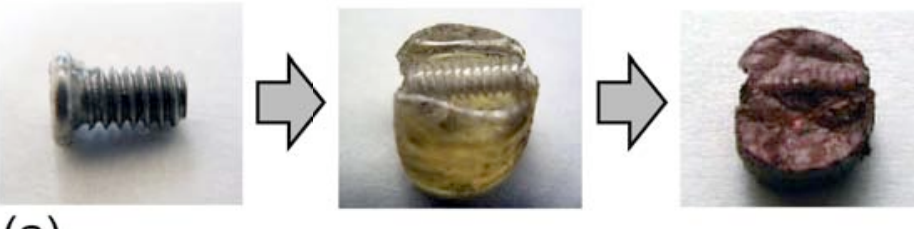

(a)

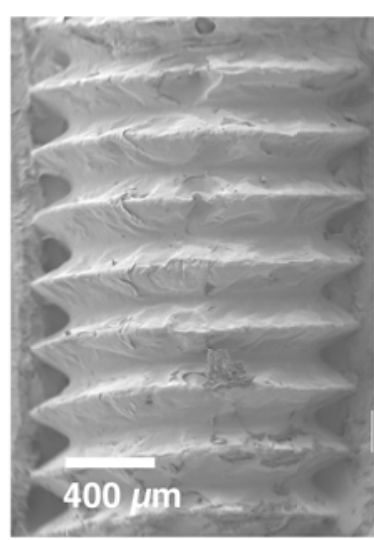

(b)

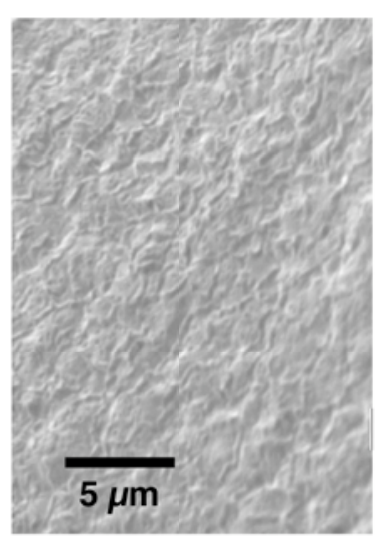

(c)

Figure 6 


\begin{tabular}{ccccc}
\hline $\begin{array}{c}\text { electroless } \\
\text { Pd plating }\end{array}$ & $\begin{array}{c}\mathrm{pH} \mathrm{6} 6) \\
(\text { mass \%) }\end{array}$ & $\begin{array}{c}\mathrm{pH} 8 \\
\text { (mass \%) }\end{array}$ & $\begin{array}{c}\mathrm{pH} \mathrm{10} \\
(\text { mass \%) }\end{array}$ & $\begin{array}{c}\mathrm{pH} \mathrm{12} \\
(\text { mass \%) }\end{array}$ \\
\hline $\mathrm{Pd}$ & 89.6 & 91.6 & 93.2 & 97.1 \\
$\mathrm{P}$ & 10.2 & 8.2 & 5.8 & 2.7 \\
$\mathrm{Fe}$ & 0.2 & $\mathrm{UDL}$ & 0.3 & 0.3 \\
$\mathrm{~S}$ & $\mathrm{UDL}$ & 0.1 & $\mathrm{UDL}$ & $\mathrm{UDL}$ \\
$\mathrm{Ni}$ & $\mathrm{UDL}$ & $\mathrm{UDL}$ & 0.1 & $\mathrm{UDL}$ \\
\hline
\end{tabular}

Table I 